\title{
HIGHER HOMOTOPY GROUPOIDS AND TODA BRACKETS
}

\author{
K.A. HARDIE, K.H. KAMPS AND R.W. KIEBOOM
}

(communicated by Ronald Brown)

\begin{abstract}
We describe a category htTop* whose objects are pointed continuous maps and whose morphisms are generated under composition by the tracks (relative homotopy classes) of homotopies. For example, if $m_{t}: h k \rightarrow *$ is a nullhomotopy then its track is a morphism from $k$ to $h$. The composition of tracks in htTop* amounts to a sharpening of the classical secondary composition operation (Toda bracket). Standard properties of the Toda bracket can be derived in this setting. Moreover we show that htTop* is itself the homotopy category of a bicategory bTop* $^{*}$ and so admits also a secondary composition operation.
\end{abstract}

\section{Introduction}

Secondary and higher order homotopy composition operations (Toda brackets) were introduced by $\mathrm{H}$. Toda $[\mathrm{T}]$ in order to construct elements of the homotopy groups of spheres as part of his 'composition method' for computing these groups. Although at first the appropriate definitions of the higher order brackets were not entirely clear, at least since the work of G. Walker [W] and M. Mori $[\mathrm{M}]$ these have been well enough understood to enable their basic properties to be determined and to permit their application to computation. Our present knowledge, however, leaves something to be desired. For example, although we have a convenient upper bound for the indeterminacy of a bracket in [W; Theorem 2] (see also [M; Proposition 4]) it is apparently not known whether this bound is the best possible.

More urgently, Baues and Dreckmann [BD] have shown, for certain full subcategories of the pointed homotopy category, that the operation of the secondary homotopy composition (i.e. the triple Toda bracket) is determined by a certain 3-dimensional class in the cohomology of the subcategory with coefficients in a natural system of abelian groups. It is reasonable to conjecture that analogous results can be proved for the higher order operations, but one is brought to a standstill when one formulates the question: in what category does there exist a characteristic cohomology class that determines (in the sense of Baues-Dreckmann) the quaternary Toda bracket?

To make progress one needs to recall another lacuna. Much of the development of algebraic topology has proceeded under the dominant influence of the nineteenth century concept of group. Although, by the 1960's, it had been recognised that the fundamental groupoid of a topological space has significant conceptual advantages over its (collection of) fundamental group(s) (cf. [Br1, Br2]) this structure has not yet fully been exploited by homotopy theorists. It is true that, since the work of Barratt [Bar] and Rutter $[\mathrm{R}]$, tracks (i.e. relative homotopy classes of homotopies) have been objects of interest in their own right, but their usual fate is to suffer the indignity of being converted into group elements by a difference construction. To come into their own they need to be seen as morphisms of a 'homotopy category' with an appropriate role. We achieve this by modifying the difference construction into a 'pasting'

Received 12 January 1999, revised 25 May 1999; published on 4 June 1999.

1991 Mathematics Subject Classification: 55Q05, 55Q35

Key words and phrases: Toda bracket, bicategory, track, double category, 2-groupoid, 2-track, Hopf invariant

(C) 1999, K.A. Hardie, K.H. Kamps and R.W. Kieboom. Permission to copy for private use granted. 
composition.

We take the view that the higher order homotopy composition operations are essentially (determined by) triple Toda brackets in a homotopy category whose morphisms are tracks of the appropriate dimension.

To clarify what we have in mind, suppose that $k: V \rightarrow U, h: U \rightarrow Z$ are pointed maps whose composite is homotopically trivial and let $m_{t}: h k \simeq *$ be a nullhomotopy. The relative homotopy class of $m_{t}$ is a 1 -track which we will denote by $\mathbf{m}$. We shall regard $\mathbf{m}$ as a morphism from $k$ to $h$ in a category whose objects are pointed maps. To understand the composition (which will be defined purely formally in section 1), note that we also regard the morphism as an oriented square

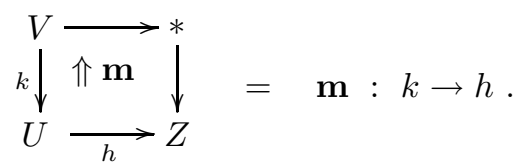

The domain and codomain arrows moving anticlockwise around the square establish its orientation. Moreover the morphism $\mathbf{m}: k \rightarrow h$ has other representations such as

$$
\begin{aligned}
& V \stackrel{k}{\longrightarrow} U \\
& \downarrow \begin{array}{l}
\Uparrow-\left.\mathbf{m}\right|_{h} \\
* \longrightarrow Z
\end{array}
\end{aligned} \quad \text { and } \quad k \uparrow \Uparrow-\left.\mathbf{m}\right|_{h}
$$

but note that here the domain and codomain arrows establish the opposite orientations and, to compensate, the tracks inhabiting the diagrams are $-\mathbf{m}$.

Suppose that $\mathbf{n}: h \rightarrow g$ is another morphism, where $g: Z \rightarrow Y$ is a map and $n_{t}: g h \simeq *$ a nullhomotopy with associated track $\mathbf{n}$. Then we define

$$
\mathbf{n} \mathbf{\Delta} \mathbf{m}=-g \cdot \mathbf{m}+k \cdot \mathbf{n}: k \rightarrow g
$$

(where $-g \cdot \mathbf{m}$ and $k \cdot \mathbf{n}$ are the relative homotopy classes $\left\{g m_{1-t}\right\}$ and $\left\{n_{t} k\right\}$ respectively), i.e. to be the track pasting

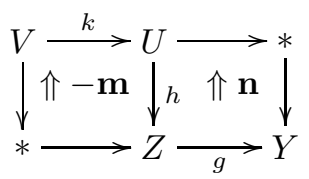

of the squares associated with $\mathbf{m}$ and $\mathbf{n}$. Note that this pasting takes place in the classical double category of track homotopy commutative squares of pointed maps. The composite morphism $\mathbf{n} \mathbf{\Delta} \mathbf{m}$ can also be identified with various oriented squares such as

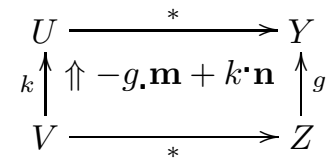

but note that the squares $(0.1)$ and $(0.2 .1)$ are of a different type. We say that the morphism $\mathbf{m}: k \rightarrow h$ is of type 1 and $\mathbf{n} \mathbf{\Delta} \mathbf{m}: k \rightarrow g$ is of type 2 . There is very little distinction between the square (0.2.1) and the track inhabiting it. With very mild abuse we blur the distinction and regard $\mathbf{n} \mathbf{\Delta} \mathbf{m}$ also as an element of the $*$-based track group $\pi^{V}(Y ; *)$ in the sense of $[\mathrm{R}]$. It is well-known that there is an isomorphism

$$
\theta: \pi^{V}(Y ; *) \stackrel{\approx}{\longrightarrow} \pi(\Sigma V, Y)
$$


Then, denoting the homotopy classes of the maps $g, h, k$ by $\beta, \gamma, \delta$ respectively, we recognise $\theta(\mathbf{n} \mathbf{\Delta} \mathbf{m}) \in \pi(\Sigma V, Y)$ as an element of the triple Toda bracket:

$$
\theta(\mathbf{n \Delta \mathbf { m }}) \in\{\beta, \gamma, \delta\} \subseteq \pi(\Sigma V, Y) .
$$

Moreover, let $f: Y \rightarrow X$ be a map and $p_{t}: f g \simeq *$ a nullhomotopy with associated track $\mathbf{p}$. More general compositions of squares such as $\mathbf{m}, \mathbf{n}, \mathbf{p}$ are defined via pasting in the double category of homotopy commutative squares. For example $\mathbf{p} \mathbf{\Delta}(\mathbf{n} \mathbf{\Delta} \mathbf{m})$ and $(\mathbf{p} \mathbf{\Delta}) \mathbf{\Delta} \mathbf{m}$ have a common definition namely the track inhabiting the pasting

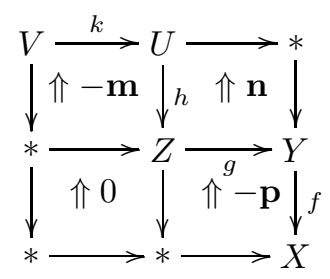

of the double category of homotopy commutative squares. Thus we get the associativity property for free. We shall see that the above instance of associativity (via the middle four interchange of the double category) translates into the bijection [T; Proposition 1.2 (iv)] between the cosets

$$
\alpha \circ\{\beta, \gamma, \delta\}=-\{\alpha, \beta, \gamma\} \circ \Sigma \delta,
$$

where $\alpha$ denotes the homotopy class of $f$.

The properties of the 'category of maps and tracks' htTop* obtained in this way are pursued further in section 1 .

To represent htTop* as a homotopy category we invoke the theory of the homotopy 2-groupoid $\mathbf{G}_{2} E$ of a Hausdorff space $E$ studied in [HKK]. By considering the case $E=X^{V}$, the (unpointed) space of pointed continuous maps from $V$ to $X$ and taking an adjoint, we obtain a 2 -groupoid $\mathbf{G}_{2}(V, X)$ whose objects are pointed maps from $V$ to $X$, whose 1-morphisms called semitracks are equivalence classes $\left\langle h_{t}\right\rangle$ of homotopies $h_{t}: V \rightarrow X$ under a relation finer than relative homotopy, and whose 2-morphisms are 2 -tracks. We then construct a bicategory bTop* whose 2-morphisms are 2-tracks and whose underlying (weak) category is similar to htTop* except that tracks $\mathbf{m}$ are replaced by semitracks $\left\langle m_{t}\right\rangle$. Finally we show that htTop* is the quotient category obtained from the underlying category of bTop* by factoring out by the homotopy relation between the 1-morphisms induced by the 2 -tracks.

Now suppose that $\mathbf{n} \mathbf{\Delta} \mathbf{m}=0$ and $\mathbf{p} \mathbf{\Lambda}=0$. Since (as we claim) our category is a homotopy category one might hope to define a triple Toda bracket $\{\mathbf{p}, \mathbf{n}, \mathbf{m}\}$ and to show that

$$
\{\mathbf{p}, \mathbf{n}, \mathbf{m}\} \subseteq\{\alpha, \beta, \gamma, \delta\} \subseteq \pi\left(\Sigma^{2} V, X\right),
$$

where the four-fold bracket refers to the classical quaternary Toda bracket. However, the underlying 'category' of bTop* is only a weak category and an extension of the classical theory of the Toda bracket is required. It is intended that this theory will be given in a paper devoted to the study of the bracket in an arbitrary bicategory and applied there to obtain the inclusion (0.6) together with other applications. Meanwhile further ramifications of htTop* , including some Hopf invariant theory involving a new formula for the classical Toda bracket are given in section 4, together with a sample application in which a (new) nontrivial Toda bracket in the homotopy groups of spheres is detected.

The authors acknowledge conversations with J.J.C. Vermeulen, comments and suggestions by R. Brown and by the referee. 


\section{The category htTop*}

We begin with some historical remarks. The notion of double category was first introduced by C. Ehresmann [E], see also [BS; S; SW]. Track homotopy commutative squares of maps have been used extensively in coherent homotopy theory. For the definition of the double category of track homotopy commutative squares in an abstract setting we refer to [KP; Chapter IV] and to $[\mathrm{S}]$.

As mentioned in the introduction the objects of htTop* are pointed continuous maps and the morphisms are generated by composing squares of the form (0.1). However, we shall need a more formal description. A morphism of the category has a type which is an integer $r$, where $0 \leqslant r \leqslant 3$. For each object (i.e. pointed map) $k$ there is an identity morphism $1_{k}: k \rightarrow k$. A morphism of type $1, \mathbf{m}: k \rightarrow h$, is only defined if $\operatorname{codomain}(k)=\operatorname{domain}(h)$ and $h k \simeq *$. Then it consists of an element of the track set $\pi^{V}(Z ; h k, *)$, in the sense of [R], where $V=\operatorname{domain}(k)$ and $Z=$ codomain $(h)$. For arbitrary maps $h: U \rightarrow Z$ and $f: Y \rightarrow X$ morphisms of type 2 and type $3, \mathbf{r}: h \rightarrow f$ are always defined. Geometrically the morphisms may be regarded as diagrams of the form

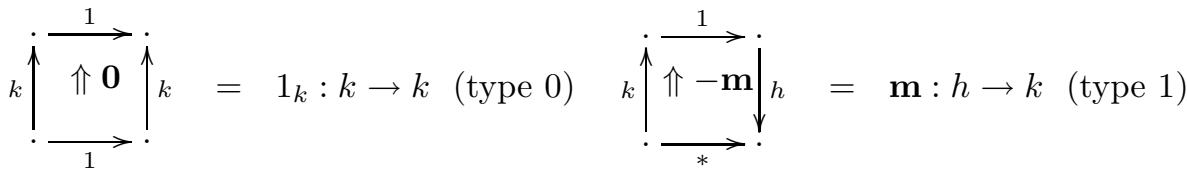

$$
\begin{aligned}
& { }_{h}^{i} \stackrel{*}{\longrightarrow \mathbf{r}} \uparrow_{*}^{\longrightarrow} f=\mathbf{r}: h \rightarrow f \quad(\text { type } 2) \quad h \prod_{\underset{*}{\longrightarrow}}^{\stackrel{*}{\longrightarrow}} ; f=\mathbf{r}: h \rightarrow f \quad \text { (type 3) }
\end{aligned}
$$

where $\mathbf{r} \in \pi^{U}(X ; *)$, corresponding to identity arrows and composites of one, two and three (respectively) morphisms of type 1 . The diagrams compose via suitable pasting in the double category of track homotopy commutative squares. Here some of the diagrams have to be turned upside down (cf. the definition of $\mathbf{n} \mathbf{\Delta} \mathbf{m}$ in (0.2)) and the positions of their bounding arrows rearranged.

The following composition formulae can be checked. Note that the interpretation is consistent with that given in the introduction.

$$
\begin{aligned}
& \mathbf{n} \mathbf{\Delta}=-g \cdot \mathbf{m}+k \cdot \mathbf{n} \quad(\mathbf{m}: k \rightarrow h, \mathbf{n}: h \rightarrow g \text { of type } 1) \\
& \mathbf{r} \mathbf{\Delta} \mathbf{m}=-k \cdot \mathbf{r} \quad(\mathbf{m}: k \rightarrow h \text { of type } 1, \mathbf{r}: h \rightarrow f \text { of type } 2 \text { or } 3) \\
& \mathbf{p} \mathbf{\Delta}=f . \mathbf{r} \quad(\mathbf{p}: g \rightarrow f \text { of type } 1, \mathbf{r}: k \rightarrow g \text { of type } 2 \text { or } 3) \\
& \mathbf{s} \mathbf{r}=0 \quad(\mathbf{r}: k \rightarrow g \text { of type } 2 \text { or } 3, \mathbf{s}: g \rightarrow e \text { is of type } 2 \text { or } 3)
\end{aligned}
$$

Although a track $\mathbf{m}$ always has an inverse $\mathbf{- m}$, note that morphisms of types $1,2,3$ are not invertible.

We denote by $\operatorname{hom}_{r}(k, f)$ the set of morphisms in htTop* of type $r$ from $k: V \rightarrow U$ to $f: Y \rightarrow X$.

1.2. Proposition. The pasting composition is associative and htTop* is a category. There exist bijective correspondences as follows.

(i) If $h k \simeq *$ then $h_{0}(k, h) \leftrightarrow \pi(\Sigma V, Z)$.

(ii) $h_{2}(k, g) \leftrightarrow \bigcup_{g_{*} \gamma=0, k^{*} \gamma=0}\{\{g\}, \gamma,\{k\}\}$.

(iii) $\operatorname{hom}_{3}(k, f) \leftrightarrow \bigcup_{f_{*} \beta=0, \beta \circ \gamma=0, k^{*} \gamma=0} f_{*}\{\beta, \gamma,\{k\}\}$. 
The first assertion of the Proposition is a consequence of the definition in terms of the double category. However we shall give a direct argument from the formal definitions in this section. We recall the well-known interchange property for 1-tracks.

1.3. Lemma. Let $k_{t}: k_{0} \simeq k_{1}: V \rightarrow U$ and $h_{t}: h_{0} \simeq h_{1}: U \rightarrow Z$ be a pair of homotopies. Then $\left(k_{0}\right) \cdot \mathbf{h}+\left(h_{1}\right) \cdot \mathbf{k}=\left(h_{0}\right) \cdot \mathbf{k}+\left(k_{1}\right) \cdot \mathbf{h}$.

Proof It is sufficient to observe that $H_{t, s}=h_{(1-s) t} k_{s t}+h_{s t+1-s} k_{s+(1-s) t}$ is a relative homotopy from $h_{t} k_{0}+h_{1} k_{t}$ to $h_{0} k_{t}+h_{t} k_{1}$.

Proof of Proposition 1.2 To check associativity of $\boldsymbol{\Delta}$ we need to examine cases. Suppose three morphisms $\mathbf{m}: k \rightarrow h, \mathbf{n}: h \rightarrow g, \mathbf{p}: g \rightarrow f$ are each of type 1. Applying (1.1) we find

$$
\mathbf{p} \mathbf{\Delta}(\mathbf{n} \mathbf{\Delta} \mathbf{m})=f .(-g \cdot \mathbf{m}+k \cdot \mathbf{n})=-f . g \cdot \mathbf{m}+f \cdot k \cdot \mathbf{n}
$$

and

$$
(\mathbf{p} \mathbf{\Delta}) \mathbf{\Delta} \mathbf{m}=-k \cdot(-f . \mathbf{n}+h \cdot \mathbf{p})=-k \cdot h \cdot \mathbf{p}+k \cdot f . \mathbf{n} .
$$

However $-f . g . \mathbf{m}=-k^{*} h \cdot \mathbf{p}$ by an application of Lemma 1.3 and, since the operators $f$. and $k$. commute, the desired equality follows.

Suppose now that $\mathbf{q}: f \rightarrow e$ is a morphism of type 1 . Then $(\mathbf{q} \mathbf{\Delta} \mathbf{p}) \mathbf{\Delta}(\mathbf{n} \mathbf{\Delta} \mathbf{m})=0$, being a composition of morphisms of type 2. Moreover $\mathbf{q} \mathbf{\Delta}(\mathbf{p} \mathbf{\Delta}(\mathbf{n} \mathbf{\Delta} \mathbf{m}))=e_{\mathbf{.}}(-f \cdot g \cdot \mathbf{m}+f \cdot k \cdot \mathbf{n})=(e f) \cdot(-g \cdot \mathbf{m}+$ $k \cdot \mathbf{n})=0$. Dually we may argue that $((\mathbf{q} \mathbf{\Delta} \mathbf{p}) \mathbf{\Delta} \mathbf{n}) \mathbf{\Delta} \mathbf{m}=0$ and hence $((\mathbf{q} \mathbf{\Delta}) \mathbf{\Delta} \mathbf{n}) \mathbf{\Delta} \mathbf{m}=$ $\mathbf{q} \Delta(\mathbf{p} \Delta(\mathbf{n} \Delta \mathbf{m}))$. We have now checked all possible associativities. Hence htTop* is a category.

In passing we may observe that the equalities (1.3.1) and (1.3.2) verify the assertion (0.5) of the introduction.

An alternative approach to the category htTop* would be to permit further morphisms of type 0 . In such an approach a morphism of type 0 from $k^{\prime}$ to $k$ is defined if $k^{\prime}$ and $k$ are homotopic maps and then it consists of a diagram of form

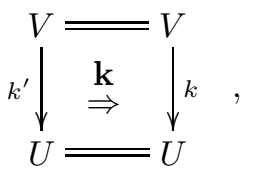

where $\mathbf{k}=\left\{k_{t}\right\}$ is the relative homotopy class of a homotopy $k_{t}: k^{\prime} \simeq k: V \rightarrow U$. The advantage of considering such morphisms of type 0 is that they become isomorphisms in the category, so that homotopic maps are necessarily isomorphic in htTop*. To avoid subsequent complication we have preferred not to adopt this approach. However, with the present definitions, homotopic maps are weakly isomorphic objects of htTop* in the following sense.

Note that the track $\mathbf{k}$ defines a function $\mathbf{k}^{*}: h o m_{1}(k, h) \rightarrow h o m_{1}\left(k^{\prime}, h\right)$ if we set

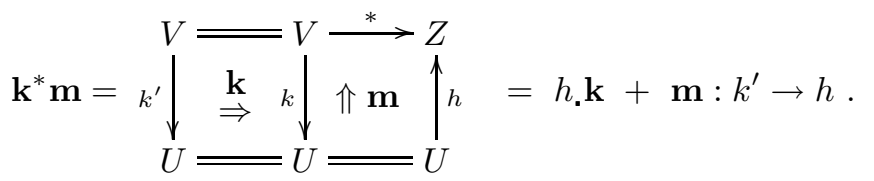

Then $\mathbf{k}^{*}$ is a bijection since $(-\mathbf{k})^{*}$ is inverse to $\mathbf{k}^{*}$. Moreover one can check, if $\mathbf{n} \mathbf{\Delta} \mathbf{m}$ is defined that

$$
\mathbf{n} \mathbf{\Delta} \mathbf{k}^{*} \mathbf{m}=\mathbf{n} \mathbf{\Delta} \mathbf{m}
$$


by considering the pasting

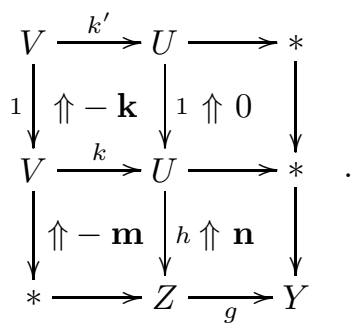

By similar arguments the remaining cases can be checked to show that the morphism sets and the composition operation in htTop* (suitably interpreted) depend only on the homotopy classes of the object maps.

It is clear that the track group $\pi^{V}(Z ; *)$ acts transitively and effectively on the set $h o m_{1}(k, h)$. With our choice of orientation, this action is on the right. If we identify $h_{0} m_{2}(k, g)$ with a subset of $\pi^{V}(Y ; *)$ via the bijection of Proposition 1.2(ii), then for $\mu \in \pi^{V}(Z ; *)$ and $\lambda \in \pi^{U}(Y ; *)$ we record

$$
\mathbf{n} . \lambda \mathbf{\Delta} \mathbf{m} \cdot \mu=-g . \mu+\mathbf{n} \mathbf{\Delta} \mathbf{m}+k \cdot \lambda .
$$

With the notation used in $(0.2)$, note that

$$
\{\beta, \gamma, \delta\}=\left\{\theta(\mathbf{n} . \lambda \mathbf{\Delta m} . \mu) \mid \lambda \in \pi^{U}(Y ; *), \mu \in \pi^{V}(Z ; *)\right\} .
$$

Then the equation (1.5) corresponds to the classical representation of $\{\beta, \gamma, \delta\}$ as a double coset.

The reader should be aware that the same track can denote many different morphisms in htTop*. For example, suppose that $k: V \rightarrow U, \lambda: U \rightarrow U^{\prime}, h: U^{\prime} \rightarrow Z, g: Z \rightarrow Y$ are maps and $m_{t}: h \lambda k \simeq *, n_{t}: g h \simeq *$ are homotopies. If $\mathbf{m}=\left\{m_{t}\right\}$ and $\mathbf{n}=\left\{n_{t}\right\}$ denote the associated tracks, then we have morphisms

$$
\lambda k \stackrel{\mathbf{m}}{\longrightarrow} h \stackrel{\mathbf{n}}{\longrightarrow} g, \quad \text { and } \quad k \stackrel{\mathbf{m}}{\longrightarrow} h \lambda \stackrel{\lambda \cdot \mathbf{n}}{\longrightarrow} g .
$$

Moreover $\mathbf{n} \mathbf{\Delta} \mathbf{m}=-g \cdot \mathbf{m}+k \cdot \lambda \cdot \mathbf{n}: \lambda k \rightarrow g$ and $\lambda \cdot \mathbf{n} \mathbf{\Delta} \mathbf{m}=-g \cdot \mathbf{m}+k \cdot \lambda \cdot \mathbf{n}: k \rightarrow g$. Note that the track parts of the respective $\boldsymbol{\Lambda}$ composites are equal.

The point is brought out well in the following Lemma.

\subsection{Lemma. Given maps}

$$
W \stackrel{\lambda}{\longrightarrow} V \stackrel{k}{\longrightarrow} U \stackrel{h}{\longrightarrow} Z \stackrel{g}{\longrightarrow} Y \stackrel{f}{\longrightarrow} X
$$

and homotopies $m_{t}: h k \lambda \simeq *, n_{t}: g h k \simeq *, p_{t}: f g h \simeq *$ then the corresponding composite morphisms in htTop*

$$
\lambda \stackrel{\mathbf{m}}{\longrightarrow} h k \stackrel{\mathbf{n}}{\longrightarrow} g \quad, \quad k \lambda \stackrel{\mathbf{m}}{\longrightarrow} h \stackrel{\mathbf{p}}{\longrightarrow} f g \quad, \quad k \stackrel{\mathbf{n}}{\longrightarrow} g h \stackrel{\mathbf{p}}{\longrightarrow} f
$$

satisfy

$$
f . \mathbf{n} \mathbf{\Delta} \mathbf{m}+\lambda \cdot \mathbf{p} \mathbf{\Delta} \mathbf{n}=\mathbf{p} \mathbf{\Delta} \mathbf{m}
$$

Proof The track equality can be verified by direct computation but the following diagrams 
offer an explanation.

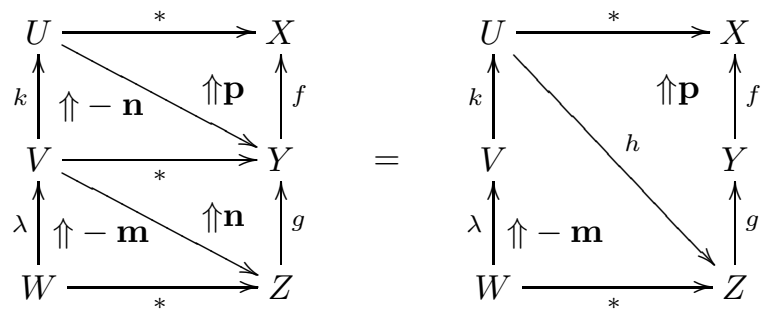

Lemma 1.6 translates into the following (possibly new) Toda bracket formula.

1.7. Proposition. Given the following sequence of spaces and homotopy classes

$$
W \stackrel{\epsilon}{\longrightarrow} V \stackrel{\delta}{\longrightarrow} U \stackrel{\gamma}{\longrightarrow} Z \stackrel{\beta}{\longrightarrow} Y \stackrel{\alpha}{\longrightarrow} X
$$

and suppose that $\alpha \beta \gamma=0, \beta \gamma \delta=0, \gamma \delta \epsilon=0$. Then

$$
\{\alpha \circ \beta, \gamma, \delta \circ \epsilon\} \subseteq \alpha \circ\{\beta, \gamma \circ \delta, \epsilon\}+\{\alpha, \beta \circ \gamma, \delta\} \circ \Sigma \epsilon .
$$

Proof Lemma 1.6 indicates that the two sides have a common element. It only remains to note that the left hand side has a smaller indeterminacy.

We shall return to the study of the category htTop* in section 4 where the emphasis will be on properties of the classical triple Toda bracket and on the form they take when expressed in this category.

\section{Properties of 2-tracks}

In this section we recall some details of the homotopy 2-groupoid $\mathbf{G}_{2} E$ of a Hausdorff space $E$ and of the 2-groupoid enrichment of the category of pointed topological spaces as given in [HKK]. For each pair of pointed spaces $X$ and $Y$ we have a 2-groupoid $\mathbf{G}_{2}(V, X)$ whose objects are pointed maps from $V$ to $X, 1$-morphisms are (equivalence classes of) pointed homotopies and whose 2-morphisms (also called 2-tracks) are (equivalence classes of) relative homotopy classes of 2-homotopies from (equivalence classes of) homotopies to homotopies.

If $p$ and $q$ are points of $E$, a path $f$ in $E$ from $p$ to $q$ is a continuous map $f: I \rightarrow E$ from the unit interval $I$ into $E$ such that $f(0)=p$ and $f(1)=q$. If $g$ is another path in $E$ from $q$ to $r$, we denote their concatenation by $g \bullet f$.

Let $f, f^{\prime}: p \simeq q$ be paths in E. A relative homotopy $f_{s}: f \simeq f^{\prime}: p \simeq q$ is a homotopy such that the initial and final points of $f$ and $f^{\prime}$ remain fixed during the homotopy. Let $f_{s}, f_{s}^{\prime}: f \simeq f^{\prime}: p \simeq q$ be two relative homotopies. We consider $f_{s}$ and $f_{s}^{\prime}$ themselves to be relatively homotopic, if they are homotopic via a homotopy $I \times I \times I \longrightarrow E$ which is constant on the boundary of $I \times I$. The relative homotopy class $\left\{f_{s}\right\}$ of $f_{s}$ is called a 2-track.

Concatenation of the relative homotopies $f_{s}$ and $g_{s}$ (defined if $f_{1}=g_{0}$ ) induces a vertical pasting operation on 2-tracks, denoted + , yielding a groupoid structure (with identities denoted 0 or $\left.0_{f}\right)$ on the set $\Pi_{2} E(p, q)$ of 2-tracks between paths in $E$ from $p$ to $q$. Similarly if $f_{s}: f \simeq$ $f^{\prime}: p \simeq q$ and $h_{s}: g \simeq g^{\prime}: q \simeq r$, the horizontal pasting of homotopies

$$
h_{s} \bullet f_{s}: g \bullet f \simeq g^{\prime} \bullet f^{\prime}: p \simeq r,
$$

obtained by concatenation of the respective paths at each stage of the homotopy, induces a corresponding operation on 2-tracks:

$$
\left(\left\{f_{s}\right\},\left\{h_{s}\right\}\right) \longrightarrow\left\{h_{s}\right\} \bullet\left\{f_{s}\right\},
$$


satisfying the interchange property

$$
\left(\left\{h_{s}\right\}+\left\{h_{s}^{\prime}\right\}\right) \bullet\left(\left\{f_{s}\right\}+\left\{f_{s}^{\prime}\right\}\right)=\left(\left\{h_{s}\right\} \bullet\left\{f_{s}\right\}\right)+\left(\left\{h_{s}^{\prime}\right\} \bullet\left\{f_{s}^{\prime}\right\}\right) .
$$

A relative homotopy $\psi_{s}: f \simeq f^{\prime}: p \simeq q$ is thin if it can be factored

$$
\psi_{s}: I \times I \stackrel{\phi_{s}}{\longrightarrow} J \stackrel{p}{\longrightarrow} E,
$$

where $J$ is a tree, $\phi_{s}: \phi \simeq \phi^{\prime}$ is a relative homotopy, $\phi$ and $\phi^{\prime}$ are paths in $J$ which (i) have the same initial and the same final points, (ii) are finitely piecewise linear and (iii) satisfy $p \phi=f$, $p \phi^{\prime}=f^{\prime}$.

The underlying groupoid $\mathbf{G} E$ of $\mathbf{G}_{2} E$ is the groupoid of $\sim$ classes of paths in $\mathrm{E}$, where $f \sim f^{\prime}$ if there exists a thin relative homotopy from $f$ to $f^{\prime}$ and where the operation $\bullet$ is induced by concatenation of paths. Let $\mathbf{N} E(p, q)$ denote the subgroupoid of $\boldsymbol{\Pi}_{2} E(p, q)$ whose morphisms are the relative homotopy classes of thin relative homotopies. Then $\mathbf{N} E(p, q)$ is a normal subgroupoid of $\boldsymbol{\Pi}_{2} E(p, q)$ and we define $\mathbf{G}_{2} E(p, q)$ to be the quotient groupoid $\boldsymbol{\Pi}_{2} E(p, q) / \mathbf{N} E(p, q)$. We use $\langle f\rangle$ to denote the $\sim$ class of a path $f$ and $0_{\langle f\rangle}$ to denote the identity 2-track in $\mathbf{G}_{2}(E,\langle f\rangle)=\mathbf{G}_{2} E(\langle f\rangle,\langle f\rangle)$. The main result of [HKK] may be stated as follows.

2.1. Proposition. The sets $\mathbf{N} E(p, q)\left(f, f^{\prime}\right)$ are singletons or empty. $\mathbf{G}_{2} E$ is a 2-groupoid with underlying groupoid $\mathbf{G} E$, 2-morphism sets $\mathbf{G}_{2}(p, q)\left(\langle f\rangle,\left\langle f^{\prime}\right\rangle\right)$ and horizontal composition

-. $\mathbf{G}_{2} E$ is functorial in $E$. For each path $f$ in $E$, there is a natural isomorphism (of abelian groups)

$$
\sigma_{\langle f\rangle}: \mathbf{G}_{2}(E,\langle f\rangle) \stackrel{\approx}{\longrightarrow} \pi_{2}(E, f(0)),
$$

where $\pi_{2}(E, f(0))$ refers to the second homotopy group of $E$ based at the point $f(0)$.

Under a systematic adjunction (applying also to the detail of the construction) the objects of $\mathbf{G}_{2} X^{V}$ and $\mathbf{G}_{2}(V, X)$ remain pointed maps from $V$ to $X$ and we have the following table of correspondences.

$$
\begin{aligned}
& \mathbf{G}_{2} X^{V} \leftrightarrow \mathbf{G}_{2}(V, X) \\
& f: I \rightarrow X^{V} \leftrightarrow f_{t}: V \rightarrow X \\
&\langle f\rangle\left.\leftrightarrow f_{t}\right\rangle \\
& f_{s}: f \simeq f^{\prime} \leftrightarrow f_{t, s}: f_{t} \simeq f_{t}^{\prime} \\
&\left\{f_{s}\right\}+\left\{g_{s}\right\} \leftrightarrow\left\{f_{t, s}\right\}+\left\{g_{t, s}\right\} \\
&\left\{f_{s}\right\} \bullet\left\{h_{s}\right\} \leftrightarrow\left\{f_{t, s}\right\} \bullet\left\{h_{t, s}\right\} \\
& \mathbf{\Pi}_{2} X^{V} \leftrightarrow \mathbf{\Pi}_{2}(V, X) \\
& \mathbf{N} X^{V} \leftrightarrow \mathbf{N}(V, X)
\end{aligned}
$$

Note that for each 1-morphism $\left\langle f_{t}\right\rangle$, with $f_{0}$ homotopic to the constant map, there is an isomorphism

$$
\sigma_{\left\langle f_{t}\right\rangle}: \mathbf{G}_{2}(V, X)\left(\left\langle f_{t}\right\rangle\right) \stackrel{\approx}{\longrightarrow} \pi\left(\Sigma^{2} V, X\right) .
$$


The elements (2-morphisms) of $\mathbf{G}_{2}(V, X)\left(\left\langle f_{t}\right\rangle,\left\langle f_{t}^{\prime}\right\rangle\right)$ are generalised cosets of $\mathbf{N}(V, X)$ determined by 2-tracks. We shall refer to them also (with some abuse) as 2-tracks and denote them by capital letters. In the following summary of their properties, we make use of the 'star operations'

$$
\left\langle f_{t}\right\rangle_{*} H=0_{\left\langle f_{t}\right\rangle} \bullet H \quad \text { and } \quad\left\langle g_{t}\right\rangle^{*} H=H \bullet 0_{\left\langle g_{t}\right\rangle},
$$

where $0_{\left\langle f_{t}\right\rangle}$ refers to the identity element in $\mathbf{G}_{2}(V, X)\left(\left\langle f_{t}\right\rangle\right)$. In the following list of properties, $c_{t}$ refers to a constant homotopy.

$$
\begin{gathered}
H+(G+F)=(H+G)+F \\
H+0=0+H=H \\
H+(-H)=0=(-H)+H \\
\left\langle h_{t}\right\rangle^{*}(-H)=-\left\langle h_{t}\right\rangle^{*} H, \quad\left\langle g_{t}\right\rangle_{*}(-H)=-\left\langle g_{t}\right\rangle_{*} H \\
\left\langle h_{t}\right\rangle^{*}(H+G)=\left\langle h_{t}\right\rangle^{*} H+\left\langle h_{t}\right\rangle^{*} G, \quad\left\langle g_{t}\right\rangle_{*}(H+G)=\left\langle g_{t}\right\rangle_{*} H+\left\langle g_{t}\right\rangle_{*} G \\
\left(\left\langle g_{t}\right\rangle \bullet\left\langle f_{t}\right\rangle\right)_{*}=\left\langle g_{t}\right\rangle_{*}\left\langle f_{t}\right\rangle_{*}, \quad\left\langle c_{t}\right\rangle_{*}=\text { identity } \\
\left(\left\langle f_{t}\right\rangle \bullet\left\langle h_{t}\right\rangle\right)^{*}=\left\langle h_{t}\right\rangle^{*}\left\langle f_{t}\right\rangle^{*}, \quad\left\langle c_{t}\right\rangle^{*}=\text { identity, }\left\langle g_{t}\right\rangle_{*}\left\langle h_{t}\right\rangle^{*}=\left\langle h_{t}\right\rangle^{*}\left\langle g_{t}\right\rangle_{*} \\
G \bullet H=\left\langle f_{t}\right\rangle^{*} G+\left\langle g_{t}^{\prime}\right\rangle_{*} H=\left\langle g_{t}\right\rangle_{*} H+\left\langle f_{t}^{\prime}\right\rangle^{*} G,
\end{gathered}
$$

in the situation

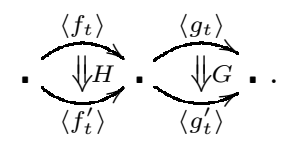

Each 2-track $H:\left\langle f_{t}\right\rangle \rightarrow\left\langle f_{t}^{\prime}\right\rangle$ has an inverse with respect to the horizontal composition denoted $H^{-1}:\left\langle f_{1-t}\right\rangle \rightarrow\left\langle f_{1-t}^{\prime}\right\rangle$. Via the equations 2.2 .1 we obtain

$$
H^{-1}=-\left\langle f_{1-t}^{\prime}\right\rangle^{*}\left\langle f_{1-t}\right\rangle_{*} H=-\left\langle f_{1-t}\right\rangle^{*}\left\langle f_{1-t}^{\prime}\right\rangle_{*} H .
$$

These properties are all standard in a 2 -groupoid. They indicate that $\mathbf{G}_{2}(V, X)$ has the structure of a track category in the sense of [Bau].

Moreover, $\mathbf{G}_{2}(V, X)$ is a bifunctor, covariant in $X$ and contravariant in $V$ : given pointed maps $k^{\prime}: V^{\prime} \rightarrow V$ and $f^{\prime}: X \rightarrow X^{\prime}$ there are associated 2- groupoid morphisms

$$
\left(k^{\prime}\right)^{\cdot}: \mathbf{G}_{2}(V, X) \rightarrow \mathbf{G}_{2}\left(V^{\prime}, X\right) \text { and } \quad f^{\prime} .: \mathbf{G}_{2}(V, X) \rightarrow \mathbf{G}_{2}\left(V, X^{\prime}\right) \text {. }
$$

We conclude this section with a brief review of interchange 2-tracks. These play a significant role in the remainder of the paper. We begin by recalling that Lemma 1.3 depends for its validity on the construction of an interchange homotopy. Specifically, suppose that $f_{t}: A \rightarrow C$ and $g_{t}: C \rightarrow B$ are homotopies. Then there is an associated 2-track, an interchange, depending only on $f_{t}$ and $g_{t}$ and inhabiting the diagram

$$
\begin{gathered}
g_{0} f_{0} \stackrel{g_{t} f_{0}}{\longrightarrow} g_{1} f_{0} \stackrel{g_{1} f_{t}}{\longrightarrow} g_{1} f_{1} \\
\|_{g_{0} f_{0} \underset{g_{0} f_{t}}{\longrightarrow}} g_{0} f_{1} \underset{g_{t} f_{1}}{\longrightarrow} g_{1} f_{1}
\end{gathered} .
$$

Various essentially equivalent versions of the interchange can be found in the literature but a rather convenient construction, via 'lens collapse', has recently been described by M. Grandis 
$[\mathrm{G}]$ : an adjoint map to $h_{t, s}$ is suggested by the following sketch.

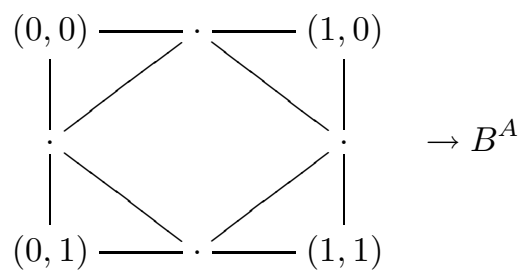

The outer square is first retracted on to the inner square by collapsing the triangles vertically on to its edges. The inner square is then mapped to $B^{A}$ by applying the adjoint of the composite 2-homotopy $g_{t} f_{s}: A \rightarrow B$. We denote the relative homotopy class of $h_{t, s}$ by $\left[g_{t}, f_{t}\right]$. A proof of the following proposition is given in $[\mathrm{HKK}]$.

2.4. Proposition. The 2-track $\left[g_{t}, f_{t}\right] \in \mathbf{G}_{2}(A, B)\left(\left\langle g_{1} f_{t} \bullet g_{t} f_{0}\right\rangle,\left\langle g_{t} f_{1} \bullet g_{0} f_{t}\right\rangle\right)$ depends only on the classes $\left\langle g_{t}\right\rangle$ and $\left\langle f_{t}\right\rangle$. The following properties hold.

(i) For a constant homotopy $c_{t}, \quad\left[g_{t}, c_{t}\right]=0=\left[c_{t}, f_{t}\right]$;

(ii) $\left[h_{t} \bullet g_{t}, f_{t}\right]=\left\langle g_{t} f_{0}\right\rangle^{*}\left[h_{t}, f_{t}\right]+\left\langle h_{t} f_{1}\right\rangle_{*}\left[g_{t}, f_{t}\right]$;

(iii) $\left[g_{t}, k_{t} \bullet f_{t}\right]=\left\langle g_{1} k_{t}\right\rangle_{*}\left[g_{t}, f_{t}\right]+\left\langle g_{0} f_{t}\right\rangle^{*}\left[g_{t}, k_{t}\right]$;

(iv) $\left[g_{1-t}, f_{t}\right]=-\left\langle g_{1-t} f_{0}\right\rangle^{*}\left\langle g_{1-t} f_{1}\right\rangle_{*}\left[g_{t}, f_{t}\right]$;

(v) $\left[g_{t}, f_{1-t}\right]=-\left\langle g_{0} f_{1-t}\right\rangle^{*}\left\langle g_{1} f_{1-t}\right\rangle_{*}\left[g_{t}, f_{t}\right]$;

(vi) Given $k: A^{\prime} \rightarrow A, f_{t}: A \rightarrow C, g_{t}: C \rightarrow B$ then $\left[g_{t}, f_{t} k\right]=k \cdot\left[g_{t}, f_{t}\right]$.

(vii) Given $f_{t}: A \rightarrow C, g_{t}: C \rightarrow B, h: B \rightarrow B^{\prime}$ then $\left[h g_{t}, f_{t}\right]=h \cdot\left[g_{t}, f_{t}\right]$.

(viii) Given $f_{t}: A \rightarrow B, g: B \rightarrow C, h_{t}: C \rightarrow D$ then $\left[h_{t} g, f_{t}\right]=\left[h_{t}, g f_{t}\right]$.

(ix) $\left[g_{t}, f_{t}\right]^{-1}=-\left[g_{1-t}, f_{1-t}\right]$.

(x) If $f_{0}=f_{1}=*$ and $g_{0}=g_{1}=*$ are trivial maps then $\left[g_{t}, f_{t}\right]=0$.

(xi) Given 2-tracks

$$
\begin{gathered}
\mu:\left\langle f_{t}\right\rangle \Longrightarrow\left\langle f_{t}^{\prime}\right\rangle: f \rightarrow f^{\prime}: A \rightarrow B, \\
\nu:\left\langle g_{t}\right\rangle \Longrightarrow\left\langle g_{t}^{\prime}\right\rangle: g \rightarrow g^{\prime}: B \rightarrow C
\end{gathered}
$$

then

$$
\left\langle g_{t} f\right\rangle^{*}\left(g^{\prime} \cdot \mu\right)+\left\langle g^{\prime} f_{t}^{\prime}\right\rangle_{*}(f \cdot \nu)+\left[g_{t}^{\prime}, f_{t}^{\prime}\right]=\left[g_{t}, f_{t}\right]+\left\langle g f_{t}\right\rangle^{*}\left(f^{\prime} \cdot \nu\right)+\left\langle g_{t}^{\prime} f^{\prime}\right\rangle_{*}(g \cdot \mu)
$$

in $\mathbf{G}_{2}(A, C)\left(\left\langle g^{\prime} f_{t} \bullet g_{t} f\right\rangle,\left\langle g_{t}^{\prime} f^{\prime} \bullet g f_{t}^{\prime}\right\rangle\right)$.

\section{A bicategory bTop*}

A bicategory is a structure consisting of objects, 1-morphisms and 2-morphisms (for details see [Bé; (1.1)(i)-(vi)]). The objects of bTop* are the same as the objects of ht Top* namely the pointed continuous maps. The 1-morphisms of bTop*, analogously to htTop* are of types 0 through 3 . They consist of diagrams of similar form to the morphisms of htTop*, the only difference being that they are inhabited by semitracks instead of tracks. Thus the morphism of type $0, k \rightarrow k$ is a diagram inhabited by the semitrack $\langle k\rangle$ of the constant homotopy. A morphism of type $1,\left\langle m_{t}\right\rangle: k \rightarrow h$, is only defined if $\operatorname{codomain}(k)=\operatorname{domain}(h)$ and $m_{t}: h k \simeq *$. For maps $h: U \rightarrow Z$ and $f: Y \rightarrow X$ morphisms of type 2 and type $3,\left\langle r_{t}\right\rangle: h \rightarrow f$ are always defined, corresponding to composites of two (respectively three) 
morphisms of type 1, however, the analogous composition operator $\boldsymbol{\Delta}$ is not associative. In contrast to the use of the plus operator for a track obtained by concatenation of homotopies as in

$$
\left\{r_{t}\right\}+\left\{q_{t}\right\}=\left\{r_{t}+q_{t}\right\}
$$

we prefer to use the bullet notation for semitracks from section 2 :

$$
\left\langle r_{t}+q_{t}\right\rangle=\left\langle q_{t}\right\rangle \bullet\left\langle r_{t}\right\rangle \text {. }
$$

Note that this gives rise to a reversal of order in certain formulae.

\subsection{Lemma.}

(i) $\left\langle n_{t}\right\rangle \boldsymbol{\Lambda}\left\langle m_{t}\right\rangle=k \cdot\left\langle n_{t}\right\rangle \bullet g .\left\langle m_{t}\right\rangle^{-1} \quad\left(\left\langle m_{t}\right\rangle: k \rightarrow h,\left\langle n_{t}\right\rangle: h \rightarrow g\right.$ of type 1)

(ii) $\left\langle r_{t}\right\rangle \mathbf{\Delta}\left\langle m_{t}\right\rangle=k \cdot\left\langle r_{t}\right\rangle^{-1} \quad\left(\left\langle m_{t}\right\rangle: k \rightarrow h\right.$ of type $1,\left\langle r_{t}\right\rangle: h \rightarrow f$ of type 2 or 3$)$

(iii) $\left\langle p_{t}\right\rangle \mathbf{\Delta}\left\langle r_{t}\right\rangle=f .\left\langle r_{t}\right\rangle \quad\left(\left\langle p_{t}\right\rangle: g \rightarrow f\right.$ of type $1,\left\langle r_{t}\right\rangle: k \rightarrow g$ of type 2 or 3$)$

(iv) $\left\langle s_{t}\right\rangle\left\langle\left\langle r_{t}\right\rangle=\left\langle *_{t}\right\rangle \quad\left(\left\langle r_{t}\right\rangle: k \rightarrow g\right.\right.$ of type 2 or $3,\left\langle s_{t}\right\rangle: g \rightarrow e$ is of type 2 or 3$)$

As in htTop*, the $\boldsymbol{\Delta}$ composition of two morphisms of type 2 or 3 is trivial (i.e. is the semitrack of the constant homotopy of the trivial map). However, in bTop* the composition of a morphism of type 3 with a morphism of type 1, yields a morphism of type 2 which (in view of the failure of associativity) need not be trivial.

In a bicategory the 2-morphisms and their composition endow each 1-morphism set with the structure of a category. In bTop*, for sets of type 0 , e.g. $\operatorname{hom}(k, k)$ the category is the trivial one with one object $1_{k}$ and an identity 2-morphism $0=0_{\langle k\rangle}$. Suppose that $\left\langle m_{t}\right\rangle,\left\langle m_{t}^{\prime}\right\rangle: k \rightarrow g$ is a parallel pair of morphisms of (the same) type greater than zero, where $k: V \rightarrow U$ and $g: Z \rightarrow Y$. Recall that the semitracks $\left\langle m_{t}\right\rangle$ and $\left\langle m_{t}^{\prime}\right\rangle$ are 1-morphisms of the 2-groupoid $\mathbf{G}_{2}(V, Y)$. We identify the 2-morphism (i.e. 2-track) sets

$$
\text { bTop }^{*}\left(\left\langle m_{t}\right\rangle,\left\langle m_{t}^{\prime}\right\rangle\right)=\mathbf{G}_{2}(V, Y)\left(\left\langle m_{t}\right\rangle,\left\langle m_{t}^{\prime}\right\rangle\right)
$$

and retain the notation + for vertical composition so that $\mathbf{b T o p} *(k, g)$ has the structure of a groupoid.

As part of the bicategory structure, for each triple $(k, h, g)$ of objects of bTop* we have to have a composition functor

$$
\mathbf{b T o p}^{*}(k, h) \times \mathbf{b T o p}^{*}(h, g) \stackrel{\boldsymbol{}}{\longrightarrow} \mathbf{b T o p} *(k, g)
$$

denoted $\left(\left\langle m_{t}\right\rangle,\left\langle n_{t}\right\rangle\right) \mapsto\left\langle n_{t}\right\rangle \mathbf{\Lambda}\left\langle m_{t}\right\rangle,(G, H) \mapsto H \boldsymbol{\Delta} G$ on 1-morphisms respectively 2-morphisms. The definition on 1-morphisms has already been given. The definition on 2-morphisms has to be given casewise. Suppose $\left\langle m_{t}\right\rangle,\left\langle m_{t}^{\prime}\right\rangle: k \rightarrow h$ and $\left\langle n_{t}\right\rangle,\left\langle n_{t}^{\prime}\right\rangle: h \rightarrow g$ are morphisms of type 1 and $M:\left\langle m_{t}\right\rangle \rightarrow\left\langle m_{t}^{\prime}\right\rangle, N:\left\langle n_{t}\right\rangle \rightarrow\left\langle n_{t}^{\prime}\right\rangle$ are 2-tracks, then we define

$$
N \mathbf{\Delta} M=k \cdot N \bullet g \cdot M^{-1} \quad(M, N \text { of type } 1)
$$

(compare with 3.1, note that the type of a 2-track is defined to be the type of its domain and codomain), where $M^{-1}:\left\langle m_{t}\right\rangle^{-1} \rightarrow\left\langle m_{t}^{\prime}\right\rangle^{-1}$ is the relative homotopy class of the 2-homotopy $m_{1-t, s}$ when $M=\left\{m_{t, s}\right\}$. In this case the functoriality is a consequence of the functoriality of $\bullet$ in the appropriate 2-groupoid.

Suppose instead that $\left\langle m_{t}\right\rangle,\left\langle m_{t}^{\prime}\right\rangle: k \rightarrow h$ are of type 1 and that $\left\langle q_{t}\right\rangle,\left\langle q_{t}^{\prime}\right\rangle: h \rightarrow f$ are of type 2 or of type 3, with given 2-tracks $M:\left\langle m_{t}\right\rangle \rightarrow\left\langle m_{t}^{\prime}\right\rangle, Q:\left\langle q_{t}\right\rangle \rightarrow\left\langle q_{t}^{\prime}\right\rangle$ then we define

$$
Q \Delta M=k^{*} Q^{-1} \quad(Q \text { of type } 2 \text { or } 3, M \text { of type } 1)
$$

(compare with 3.1). Similarly if $\left\langle u_{t}\right\rangle,\left\langle u_{t}^{\prime}\right\rangle: k \rightarrow g$ are of type 2 or of type 3 and $\left\langle p_{t}\right\rangle,\left\langle p_{t}^{\prime}\right\rangle: g \rightarrow f$ are of type 1 , we define

$$
P \mathbf{\Delta} U=f . U \quad(P \text { of type } 1, U \text { of type } 2 \text { or } 3)
$$


(compare with 3.1).

We also define $Q \mathbf{\Delta} U=0,(Q, U$ of type 2 or 3$)$ and $H \mathbf{\Delta} 0=H, 0 \mathbf{\Delta} H=H \quad(0$ of type 0$)$.

The functoriality of $\boldsymbol{\Delta}$ is a consequence of the following interchange law for $\boldsymbol{\Delta}$ which can be checked by considering cases:

$$
\left(G+G^{\prime}\right) \mathbf{\Delta}\left(F+F^{\prime}\right)=(G \mathbf{\Delta} F)+\left(G^{\prime} \mathbf{\Delta} F^{\prime}\right),
$$

where $G:\left\langle g_{t}\right\rangle \rightarrow\left\langle g_{t}^{\prime}\right\rangle, G^{\prime}:\left\langle g_{t}^{\prime}\right\rangle \rightarrow\left\langle g_{t}^{\prime \prime}\right\rangle, F:\left\langle f_{t}\right\rangle \rightarrow\left\langle f_{t}^{\prime}\right\rangle, F^{\prime}:\left\langle f_{t}^{\prime}\right\rangle \rightarrow\left\langle f_{t}^{\prime \prime}\right\rangle$ and $\left\langle g_{t}\right\rangle,\left\langle g_{t}^{\prime}\right\rangle,\left\langle g_{t}^{\prime \prime}\right\rangle:$ $h \rightarrow g,\left\langle f_{t}\right\rangle,\left\langle f_{t}^{\prime}\right\rangle,\left\langle f_{t}^{\prime \prime}\right\rangle: k \rightarrow h$.

In a bicategory, for each quadruple of objects $(k, h, g, f)$ there are required to be associativity isomorphisms: given $\left\langle m_{t}\right\rangle: k \rightarrow h,\left\langle n_{t}\right\rangle: h \rightarrow g$ and $\left\langle p_{t}\right\rangle: g \rightarrow f$, we need a natural isomorphism

$$
A_{p, n, m}:\left\langle p_{t}\right\rangle \mathbf{\Lambda}\left(\left\langle n_{t}\right\rangle \mathbf{\Lambda}\left\langle m_{t}\right\rangle\right) \Longrightarrow\left(\left\langle p_{t}\right\rangle \mathbf{\Delta}\left\langle n_{t}\right\rangle\right) \mathbf{\Delta}\left\langle m_{t}\right\rangle .
$$

Moreover these isomorphisms have to satisfy the following

(AC) Associativity coherence: Given

$$
\left\langle m_{t}\right\rangle: k \rightarrow h,\left\langle n_{t}\right\rangle: h \rightarrow g,\left\langle p_{t}\right\rangle: g \rightarrow f,\left\langle q_{t}\right\rangle: f \rightarrow e
$$

then the following diagram commutes.

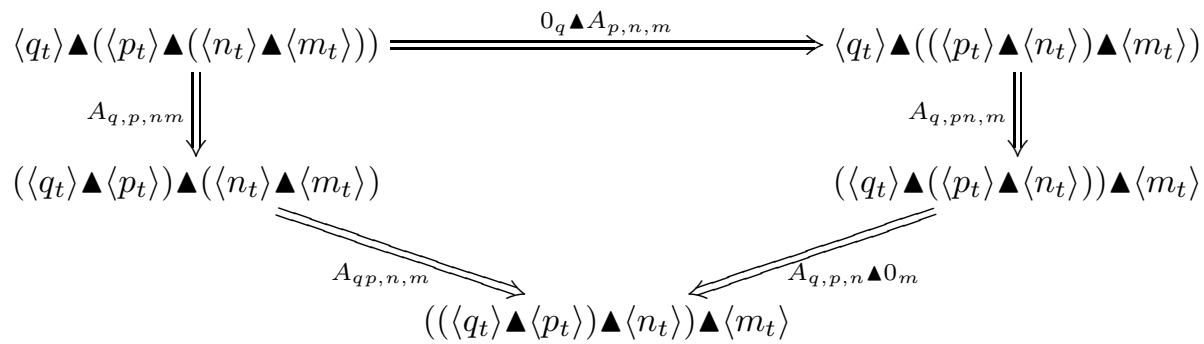

It is necessary to consider cases for the definition of $A_{p, n, m}$.

If $\left\langle m_{t}\right\rangle: k \rightarrow h,\left\langle n_{t}\right\rangle: h \rightarrow g,\left\langle p_{t}\right\rangle: g \rightarrow f$ are morphisms of type 1 , note that

$\left\langle p_{t}\right\rangle \mathbf{\Delta}\left(\left\langle n_{t}\right\rangle \mathbf{\boldsymbol { \Delta }}\left\langle m_{t}\right\rangle\right)=f . k^{*}\left\langle n_{t}\right\rangle \bullet f . g \cdot\left\langle m_{1-t}\right\rangle$ and $\left(\left\langle p_{t}\right\rangle \mathbf{\Lambda}\left\langle n_{t}\right\rangle\right) \mathbf{\Delta}\left\langle m_{t}\right\rangle=k^{*} f \cdot\left\langle n_{t}\right\rangle \bullet k^{*} h^{*}\left\langle p_{1-t}\right\rangle$, so we may define

$$
A_{p, n, m}=-\left\langle f . k^{*} n_{t}\right\rangle_{*}\left[p_{t}, m_{t}\right]^{-1} \in \mathbf{G}_{2}(V, X)\left(f . k^{*}\left\langle n_{t}\right\rangle \bullet f_{.} g .\left\langle m_{1-t}\right\rangle, f . k^{*}\left\langle n_{t}\right\rangle \bullet k^{*} h^{*}\left\langle p_{1-t}\right\rangle\right) .
$$

If $\left\langle m_{t}\right\rangle$ is of type 2 or 3 , with $\left\langle n_{t}\right\rangle$ and $\left\langle p_{t}\right\rangle$ of type 1 then $\left\langle p_{t}\right\rangle \mathbf{\Lambda}\left(\left\langle n_{t}\right\rangle \mathbf{\Delta}\left\langle m_{t}\right\rangle\right)=f . g .\left\langle m_{t}\right\rangle$ and $\left(\left\langle p_{t}\right\rangle \mathbf{\Delta}\left\langle n_{t}\right\rangle\right) \mathbf{\Delta}\left\langle m_{t}\right\rangle=\left\langle *_{t}\right\rangle=* *\left\langle p_{t}\right\rangle$, so we may define

$$
A_{p, n, m}=-\left[p_{t}, m_{t}\right] .
$$

Similarly if $\left\langle p_{t}\right\rangle$ is of type 2 or 3 with $\left\langle n_{t}\right\rangle$ and $\left\langle m_{t}\right\rangle$ of type 1 then $\left\langle p_{t}\right\rangle \mathbf{\Lambda}\left(\left\langle n_{t}\right\rangle\left\langle m_{t}\right\rangle\right)=\left\langle *_{t}\right\rangle=$ *. $\left\langle m_{t}\right\rangle$ and $\left(\left\langle p_{t}\right\rangle \mathbf{\Lambda}\left\langle n_{t}\right\rangle\right) \mathbf{\Lambda}\left\langle m_{t}\right\rangle=k \cdot h^{*}\left\langle p_{t}\right\rangle$ and so we may again define $A_{p, n, m}$ via 3.3.2.

If $\left\langle n_{t}\right\rangle$ is of type 2 or 3 , with $\left\langle m_{t}\right\rangle$ and $\left\langle p_{t}\right\rangle$ of type 1 then $\left\langle p_{t}\right\rangle \boldsymbol{\Lambda}\left(\left\langle n_{t}\right\rangle \boldsymbol{\Lambda}\left\langle m_{t}\right\rangle\right)=\left(\left\langle p_{t}\right\rangle \mathbf{\Lambda}\left\langle n_{t}\right\rangle\right) \boldsymbol{\Lambda}\left\langle m_{t}\right\rangle=$ $f . k \cdot\left\langle n_{1-t}\right\rangle$, so that we may define $A_{p, n, m}=0$.

If two or more of $\left\langle m_{t}\right\rangle,\left\langle n_{t}\right\rangle$ and $\left\langle p_{t}\right\rangle$ are of types 2 or three, we define $A_{p, n, m}=0$.

Finally, if one (or more) of $\left\langle m_{t}\right\rangle,\left\langle n_{t}\right\rangle$ and $\left\langle p_{t}\right\rangle$ is an identity element we define $A_{p, n, m}=0$.

3.4. Proposition. The associativity coherence (AC) holds in bTop*.

Proof Case 1: $\left\langle m_{t}\right\rangle: k \rightarrow h,\left\langle n_{t}\right\rangle: h \rightarrow g,\left\langle p_{t}\right\rangle: g \rightarrow f,\left\langle q_{t}\right\rangle: f \rightarrow e$ of type 1.

We have

$$
0_{q} \mathbf{\Lambda} A_{p, n, m}+A_{q, p n, m}+A_{q, p, n} \mathbf{\Delta} 0_{m}=-e \cdot\left\langle f . k^{*} n_{t}\right\rangle_{*}\left[p_{t}, m_{t}\right]^{-1}+0-k^{*}\left(\left\langle e . h \cdot p_{1-t}\right\rangle^{*}\left[q_{t}, n_{t}\right]\right)
$$


and

$A_{q, p, n m}+A_{q p, n, m}=-\left[q_{t}, n_{t} k \bullet g m_{1-t}\right]-\left[q_{t} g \bullet e p_{1-t}, m_{t}\right]$

$=-\left\langle e f g m_{1-t}\right\rangle^{*}\left[q_{t}, n_{t} k\right]-\left[q_{t}, g m_{1-t}\right]-\left[e p_{1-t}, m_{t}\right]-\left\langle e p_{1-t} h k\right\rangle^{*}\left[q_{t}, g m_{t}\right]$.

Since $-\left[q_{t}, g m_{1-t}\right]=\left\langle e f g m_{1-t}\right\rangle^{*}\left[q_{t}, g m_{t}\right]$ and $-\left[e p_{1-t}, m_{t}\right]=\left\langle e p_{1-t} h k\right\rangle^{*}\left[e p_{t}, m_{t}\right]$, it follows that

$$
-\left(0_{q} \boldsymbol{\Lambda} A_{p, n, m}+A_{q, p n, m}+A_{q, p, n} \mathbf{\Delta} 0_{m}\right)+A_{q, p, n m}+A_{q p, n . m}
$$

is the composite 2-track contained in the diagram:

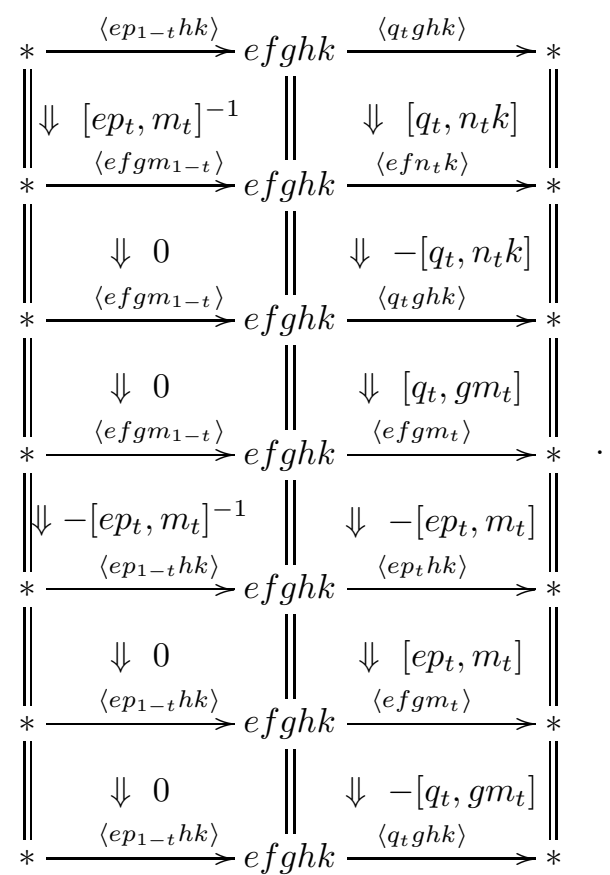

Note that the 2-tracks in the cells of the fourth row (which cancel by horizontal composition) have been inserted deliberately to facilitate cancellation of the remaining 2-tracks that originate from the expansion of the expression. In view of the interchange property for 2-tracks, we may add first the 2-tracks in the separate columns. However these cancel achieving the desired result.

Case 2: $\left\langle m_{t}\right\rangle$ of type 2, $\left\langle q_{t}\right\rangle,\left\langle n_{t}\right\rangle,\left\langle p_{t}\right\rangle$ of type 1 .

In this case we have $0_{q} \boldsymbol{\Lambda} A_{p, n, m}=-\left[e \cdot p_{t}, m_{t}\right], A_{q, p, n m}=-\left[g^{*} q_{t}, m_{t}\right]$ but the other three arrows of the coherence diagram are zero 2-tracks. Applying Proposition $2.4(\mathrm{x})$, we have $\left[e . p_{t} \bullet g^{*} q_{1-t}, m_{t}\right]=0$, which implies $\left[e . p_{t}, m_{t}\right]=\left[g^{*} q_{t}, m_{t}\right]$.

Case 3: $\left\langle p_{t}\right\rangle$ of type $2,\left\langle q_{t}\right\rangle,\left\langle n_{t}\right\rangle,\left\langle m_{t}\right\rangle$ of type 1 .

Case 3 is similar to Case 2.

Case 4: $\left\langle n_{t}\right\rangle$ of type $2,\left\langle p_{t}\right\rangle,\left\langle q_{t}\right\rangle,\left\langle m_{t}\right\rangle$ of type 1 .

In this case the arrows $0_{q} \boldsymbol{\Lambda} A_{p, n, m}, A_{q, p n, m}$ and $A_{q p, n, m}$ reduce to identity 2-tracks, but $A_{q, p, n} \mathbf{\Delta} 0_{m}$ and $A_{q, p, n m}$ are both equal to $-k \cdot\left[q_{t}, n_{1-t}\right]$.

Case 5: $\left\langle p_{t}\right\rangle$ of type $2,\left\langle q_{t}\right\rangle,\left\langle n_{t}\right\rangle,\left\langle m_{t}\right\rangle$ of type 1 .

Case 5 is similar to Case 4 .

Case 6: At least two semitracks are of types 2 or 3 .

Suppose that $\left\langle n_{t}\right\rangle$ and $\left\langle m_{t}\right\rangle$ are of type at least 2. Then, by the definition of $A_{p, n, m}, 0_{q} \mathbf{\Lambda} A_{p, n, m}$, 
$A_{q, p n, m}$ and $A_{q p, n, m} \mathbf{\Lambda}$ are zero 2-tracks. Since $\left\langle n_{t}\right\rangle \mathbf{\Delta}\left\langle m_{t}\right\rangle$ is trivial, $A_{q, p, n m}=-\left[q_{t}, *_{t}\right]=0$ and $A_{q, p, n} \mathbf{\Delta} 0_{m}=0$, by the definition of $\boldsymbol{\Lambda}$. The remaining cases (including those that arise when one or more of the 1-morphisms are identities) may now be checked via similar arguments, completing the proof of Proposition 3.4.

There is one further requirement in a bicategory that needs to be mentioned. For each pair of objects of $\mathbf{b T o p}^{*}, k$ and $g$ we need two natural isomorphisms

$$
\lambda: 1_{k} \mathbf{\Lambda}\left\langle m_{t}\right\rangle \Longrightarrow\left\langle m_{t}\right\rangle, \quad \rho:\left\langle m_{t}\right\rangle \mathbf{\Delta} 1_{g} \Longrightarrow\left\langle m_{t}\right\rangle,
$$

where $\left\langle m_{t}\right\rangle: k \rightarrow g$ is a 1-morphism. We may choose $\lambda$ and $\rho$ to be identity 2-tracks. It is then clear that these satisfy the following

(IC) Identity coherence: Let $\left\langle m_{t}\right\rangle: k \rightarrow g$ and $\left\langle p_{t}\right\rangle: g \rightarrow q$ be 1-morphisms of bTop*. Then the following diagram commutes.

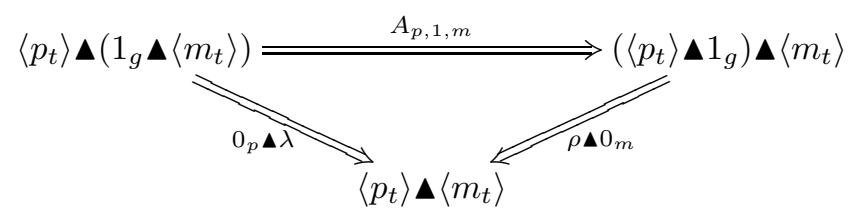

We may now define the homotopy relation in bTop*. Let $\left\langle m_{t}\right\rangle,\left\langle m_{t}^{\prime}\right\rangle: k \rightarrow g$ be 1-morphisms of the same type. Then $\left\langle m_{t}\right\rangle$ and $\left\langle m_{t}^{\prime}\right\rangle$ are homotopic, denoted

$$
\left\langle m_{t}\right\rangle \simeq\left\langle m_{t}^{\prime}\right\rangle: k \rightarrow g
$$

if and only if there exists a 2-morphism from $\left\langle m_{t}\right\rangle$ to $\left\langle m_{t}^{\prime}\right\rangle$. We have the following:

3.5. Theorem. bTop* is a bicategory, $\simeq$ is a natural equivalence relation in bTop* $^{*}$ with quotient category htTop*.

Proof We have already verified the first assertion.

If $\left\langle m_{t}\right\rangle \simeq\left\langle m_{t}^{\prime}\right\rangle$ and $\left\langle q_{t}\right\rangle \simeq\left\langle q_{t}^{\prime}\right\rangle$ and if $\operatorname{codomain}\left(\left\langle m_{t}\right\rangle\right)=\operatorname{domain}\left(\left\langle q_{t}\right\rangle\right)$ then we have to show that $\left\langle q_{t}\right\rangle \mathbf{\mathbf { \Delta }}\left\langle m_{t}\right\rangle \simeq\left\langle q_{t}^{\prime}\right\rangle\left\langle\left\langle m_{t}^{\prime}\right\rangle\right.$, regardless of the types of $\left\langle m_{t}\right\rangle$ and $\left\langle q_{t}\right\rangle$. Suppose that $\left\langle m_{t}\right\rangle,\left\langle m_{t}^{\prime}\right\rangle: k \rightarrow h$ and $\left\langle q_{t}\right\rangle,\left\langle q_{t}^{\prime}\right\rangle: h \rightarrow g$ are morphisms. and let $G \in \mathbf{b T o p}^{*}\left(\left\langle m_{t}\right\rangle,\left\langle m_{t}^{\prime}\right\rangle\right), H \in$ bTop* $^{*}\left(\left\langle q_{t}\right\rangle,\left\langle q_{t}^{\prime}\right\rangle\right)$ be 2 -tracks. We need merely note that $H \mathbf{\Delta} G \in$ bTop* $^{*}\left(\left\langle q_{t}\right\rangle \mathbf{\Lambda}\left\langle m_{t}\right\rangle,\left\langle q_{t}^{\prime}\right\rangle \mathbf{\Lambda}\left\langle m_{t}^{\prime}\right\rangle\right)$.

In view of the existence of the 2-tracks $A_{p, n, m}$, the composition $\boldsymbol{\Delta}$ in the underlying weak category of bTop* is associative up to homotopy. It follows that the homotopy category obtained by factoring out by the homotopy relation is isomorphic with htTop*, giving rise to a quotient functor

$$
p: \text { bTop }^{*} \rightarrow \text { htTop }^{*}
$$

\section{Suspension and Hopf invariant}

We show that the (reduced) suspension endofunctor $\Sigma$ of Top* gives rise to an endofunctor of htTop*. If $\mathbf{m}: k \rightarrow h$ is a morphism of type 1 and $\mathbf{m}=\left\{m_{t}\right\}$, then $\Sigma m_{t}: \Sigma h \Sigma k \simeq *$ is a nullhomotopy of $(\Sigma h)(\Sigma k)$ and we set $\Sigma \mathbf{m}=\left\{\Sigma m_{t}\right\}: \Sigma k \rightarrow \Sigma h$. If $\mathbf{s}: k \rightarrow g$ is a morphism of type 2 or 3 and $\mathbf{s}=\left\{s_{t}\right\}$, where $s_{t}: * \simeq *$, then we set $\Sigma \mathbf{s}=\left\{\Sigma s_{t}\right\}$ to be the corresponding morphism of type 2 or 3 from $\Sigma k$ to $\Sigma g$.

4.1. Proposition. As defined, $\Sigma$ is an endofunctor of htTop*.

Proof Suppose that $\mathbf{m}: k \rightarrow h$ and $\mathbf{n}: h \rightarrow g$ are morphisms of type 1 . Then

$$
\Sigma \mathbf{n} \mathbf{\Delta} \mathbf{m}=-(\Sigma g) .(\Sigma \mathbf{m})+(\Sigma k)^{\cdot}(\Sigma \mathbf{n})=-\Sigma(g \cdot \mathbf{m})+\Sigma(k \cdot \mathbf{n})=\Sigma(\mathbf{n} \mathbf{\Delta} \mathbf{m}) .
$$

The argument in the remaining cases is similar. 
The good behaviour of suspension with respect to $\boldsymbol{\Delta}$ may be contrasted with the following formula for morphisms of type 1

$$
\theta(\Sigma \mathbf{n} \mathbf{\Delta} \Sigma \mathbf{m})=-\Sigma \theta(\mathbf{n} \mathbf{\Delta} \mathbf{m}) .
$$

Here the change of sign arises through an interchange of suspension coordinates giving rise to the classical (cf. [T; Proposition 1.3])

$$
-E\{\beta, \gamma, \delta\} \subseteq\{E \beta, E \gamma, E \delta\} .
$$

In similar vein we can recognise the following analog of the formula (0.3) involving Toda's subscript bracket.

$$
\theta(\mathbf{n} \mathbf{\Sigma} \mathbf{m}) \in\{\beta, \Sigma \gamma, \Sigma \delta\}_{1}
$$

We now turn to formulae involving the Hopf-James invariant. Recall that the suspension functor $\Sigma$ is left adjoint in Top* to the loop functor $\Omega$ and that for a large class of spaces $X$ a James space $X_{\infty}$ and an associated homotopy equivalence can be defined [J]

$$
X_{\infty} \rightarrow \Omega \Sigma X
$$

inducing a canonical isomorphism

$$
\Omega_{1}: \pi(\Sigma V, \Sigma Y) \rightarrow \pi\left(V, Y_{\infty}\right) .
$$

The Hopf-James invariant is a homomorphism

$$
H=\Omega_{1}^{-1} j_{X} \Omega_{1}: \pi(\Sigma V, \Sigma X) \rightarrow \pi(\Sigma V, \Sigma(X \wedge X))
$$

induced (via the homotopy equivalence (4.2) and the $\Sigma-\Omega$ adjunction) by a James map

$$
j_{X}: X_{\infty} \rightarrow(X \wedge X)_{\infty} .
$$

Moreover there is an inclusion map $i_{X}: X \rightarrow X_{\infty}$ with the property that the composition $j_{X} i_{X}=*$. It follows that the constant homotopy of the trivial map defines a track $\mathbf{H}_{X}$ and a morphism

$$
\mathbf{H}_{X}: i_{X} \rightarrow j_{X}
$$

in htTop* which has many useful properties. For example we recall from [H; section 3] that there is a partially exact sequence

$$
\rightarrow \pi(\Sigma Y, X) \stackrel{\Sigma}{\longrightarrow} \pi\left(\Sigma^{2} Y, \Sigma X\right) \stackrel{H}{\longrightarrow} \pi\left(\Sigma^{2} Y, \Sigma(X \wedge X)\right) \stackrel{\Delta^{\leftarrow}}{\longleftarrow} \pi(Y, X) \longrightarrow
$$

which can be regarded as a generalised form of the $E H \Delta$ sequence [T]. Here $\Delta^{\leftarrow}$ is a partial function (defined with a degree of indeterminacy) via a Toda bracket

$$
\Delta^{\leftarrow}(\mu)=-\Omega_{1}^{-1}\left\{{ }^{\circ}\left\{j_{X}\right\},\left\{i_{X}\right\}, \mu\right\} \subseteq \pi\left(\Sigma^{2} V, \Sigma(X \wedge X)\right),
$$

where the little circle decorating the bracket indicates that only the preferred (trivial) nullhomotopy of $j_{X} i_{X}$ is permitted, thus reducing the indeterminacy. For the cases in which the $\Delta$ operator is defined, $\Delta^{\leftarrow}$ coincides with $\Delta^{-1}$. Note that in terms of the $\boldsymbol{\Delta}$ composition the definition can be restated

$$
\Delta^{\leftarrow}(\mu)=-\Omega_{1}^{-1} \theta\left(\mathbf{H}_{X} \mathbf{\Delta q}\right),
$$

where $\mathbf{q}$ is the track of a nullhomotopy of a representative of $i_{X} . \mu$.

As an illustrative application we indicate a proof of the following result due to Toda, cf. [T; Proposition 2.6].

4.3. Proposition. Suppose that $\alpha \in \pi(Y, X), \beta \in \pi(Z, Y)$ and $\gamma \in \pi(U, Z)$ satisfy $\Sigma(\alpha \circ \beta)=$ 0 and $\beta \circ \gamma=0$ then

$$
H\{\Sigma \alpha, \Sigma \beta, \Sigma \gamma\}_{1}=-\Delta^{\leftarrow}(\alpha \circ \beta) \circ \Sigma^{2} \gamma .
$$


Proof Suppose that $\mathbf{n}: h \rightarrow g$ and $\mathbf{p}: \Sigma g \rightarrow \Sigma f$ be morphisms of htTop*, where $h, g$ and $f$ are representatives of $\gamma, \beta$ and $\alpha$ respectively. According to Toda, [T; 2.4 and Proposition $1.3]$

$$
H\{\Sigma \alpha, \Sigma \beta, \Sigma \gamma\}_{1}=-H \Omega_{1}^{-1}\left\{i_{X} f, g, h\right\}=-\Omega_{1}^{-1} j_{X} \cdot\left\{i_{X} f, g, h\right\} .
$$

Now a typical element of $j_{X} \cdot\left\{i_{X} f, g, h\right\}$ is of the form $\theta j_{X} \cdot(\overline{\mathbf{p}} \mathbf{\Lambda})$, where $\overline{\mathbf{p}}: g \rightarrow i_{X} f$ and $\mathbf{n}: h \rightarrow g$, but

$$
j_{X} \cdot(\overline{\mathbf{p}} \mathbf{\Delta})=\mathbf{H}_{X} \mathbf{\Delta}(\overline{\mathbf{p}} \mathbf{\Delta} \mathbf{n})=\left(\mathbf{H}_{X} \mathbf{\Delta} \overline{\mathbf{p}}\right) \mathbf{\Delta} \mathbf{n}=-h \cdot\left(\mathbf{H}_{X} \mathbf{\Delta} \overline{\mathbf{p}}\right)
$$

Hence $-\Omega_{1}^{-1} \theta j_{X} \cdot(\overline{\mathbf{p}} \mathbf{\Delta} \mathbf{n})=\Omega_{1}^{-1} \theta h^{\cdot}\left(\mathbf{H}_{X} \mathbf{\Delta} \overline{\mathbf{p}}\right) \in-\Delta^{\leftarrow}(\alpha \circ \beta) \circ \Sigma^{2} \gamma$, as required. The proof may be completed by the observation, as in $[\mathrm{H}]$, that the two sides of the equality have the same indeterminacy.

Note that the essential mechanism of the proof is the associativity of the $\boldsymbol{\Delta}$ composition.

It is possible to define a dual subscript bracket for which a Hopf invariant formula can be proved. This formula does not seem to be present in the literature although a hint of its likely existence can be found in [HK] since it is related to the homotopy pair bracket formula [HK; Proposition 3.10(i)] and can be obtained from it by appropriate specialisation. The proof given below in terms of the $\boldsymbol{\Lambda}$ composition uses the equality in Lemma 1.6.

Let $h: U \rightarrow Z, g: Z \rightarrow Y$ and $f: Y \rightarrow X$ be representatives of the classes $\gamma, \beta$ and $\alpha$ respectively and suppose that they satisfy $\alpha \circ \beta=0$ and $\Sigma(\beta \circ \gamma)=0$. Then there exist morphisms in htTop*, $\mathbf{n}: \Sigma h \rightarrow \Sigma g$ and $\mathbf{p}: g \rightarrow f$ and we define

$$
{ }_{1}\{\Sigma \alpha, \Sigma \beta, \Sigma \gamma\}=\{\theta(\Sigma \mathbf{p} \mathbf{\Delta}) \mid \mathbf{n}: \Sigma h \rightarrow \Sigma g, \mathbf{p}: g \rightarrow f\} .
$$

4.4.1. Proposition. $H\left({ }_{1}\{\Sigma \alpha, \Sigma \beta, \Sigma \gamma\}\right)=\Sigma^{2}(\alpha \wedge \alpha) \circ \Delta^{\leftarrow}(\beta \circ \gamma)$.

Proof Consider the sequence of spaces and maps

$$
U \stackrel{h}{\longrightarrow} Z \stackrel{g}{\longrightarrow} Y \stackrel{i_{Y}}{\longrightarrow} Y_{\infty} \stackrel{f_{\infty}}{\longrightarrow} X_{\infty} \stackrel{j_{X}}{\longrightarrow}(X \wedge X)_{\infty}
$$

and note that this sequence satisfies the conditions of Lemma 1.6. It follows that there exist morphisms

$$
h \stackrel{\overline{\mathbf{n}}}{\longrightarrow} i_{Y} g \stackrel{i_{X} \cdot \overline{\mathbf{p}}}{\longrightarrow} f_{\infty} \quad g h \stackrel{\overline{\mathbf{n}}}{\longrightarrow} i_{Y} \stackrel{f \cdot \mathbf{H}_{X}}{\longrightarrow} j_{X} f_{\infty} \quad g \stackrel{i_{X} \cdot \overline{\mathbf{p}}}{\longrightarrow} f_{\infty} i_{Y} \stackrel{f \cdot \mathbf{H}_{X}}{\longrightarrow} j_{X} .
$$

By Lemma 1.6 we have

$$
j_{X} \cdot\left(i_{X} \cdot \overline{\mathbf{p}} \mathbf{\mathbf { n }}\right)+h^{\cdot}\left(f \cdot \mathbf{H}_{X} \mathbf{\Delta} i_{X} \cdot \overline{\mathbf{p}}\right)=\left(f \cdot \mathbf{H}_{X}\right) \mathbf{\Delta} \overline{\mathbf{n}} .
$$

Applying $\Omega_{1}^{-1} \theta$ to the terms of the above equality we obtain firstly

$$
\Omega_{1}^{-1} \theta j_{X} \cdot\left(i_{X} \overline{\mathbf{p}} \mathbf{\Delta} \overline{\mathbf{n}}\right) \in-H_{1}\{\Sigma \alpha, \Sigma \beta, \Sigma \gamma\} .
$$

Via the commutativity in

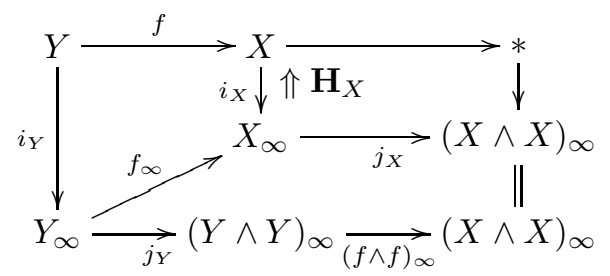

we have $f \cdot \mathbf{H}_{X}=(f \wedge f)_{\infty} \cdot \mathbf{H}_{Y}$ and hence

$$
\Omega_{1}^{-1} \theta\left(f \cdot \mathbf{H}_{X} \Delta \overline{\mathbf{n}}\right)=\Omega_{1}^{-1} \theta(f \wedge f)_{\infty} \cdot \mathbf{H}_{Y} \Delta \overline{\mathbf{n}} \in-\Sigma^{2}(\alpha \wedge \alpha) \circ \Delta^{\leftarrow}(\beta \circ \gamma) .
$$


Finally, since $i_{X} f=f_{\infty} i_{Y}$, the composite morphisms

$$
g \stackrel{i_{X} \cdot \overline{\mathbf{p}}}{\longrightarrow} f_{\infty} i_{Y} \stackrel{f \cdot \mathbf{H}_{X}}{\longrightarrow} j_{X} \quad \text { and } \quad f g \stackrel{i_{X} \cdot \overline{\mathbf{p}}}{\longrightarrow} i_{X} \stackrel{\mathbf{H}_{X}}{\longrightarrow} j_{X}
$$

contain equal tracks. Hence

$$
\Omega_{1}^{-1} \theta h \cdot\left(f \cdot \mathbf{H}_{X} \Delta i_{X} \cdot \overline{\mathbf{p}}\right)=h^{\cdot}\left(\mathbf{H}_{X} \Delta i_{X} \cdot \overline{\mathbf{p}}\right) \in-\Delta_{1}^{\leftarrow}(\alpha \circ \beta) \circ \Sigma^{2} \gamma .
$$

However, since $\alpha \circ \beta=0$ this last term belongs to the common indeterminacy of the two sides of the desired equality, which completes the proof.

We conclude with an example of a bracket whose component classes are elements of homotopy groups of spheres and which can be shown to be non-trivial via an application of Proposition 4.4.1. With the notation used in Toda's book [T], we may check (Chapter 5) that $\nu_{6} \circ \eta_{9}=0$ and $8 \iota_{5} \circ \nu_{5}=0$. Hence the bracket

$$
{ }_{1}\left\{8 \iota_{6}, \nu_{6}, \eta_{9}\right\} \subseteq \pi_{11}\left(S^{6}\right)
$$

is defined. We have

4.5. Proposition. $32\left[\iota_{6}, \iota_{6}\right] \in{ }_{1}\left\{8 \iota_{6}, \nu_{6}, \eta_{9}\right\} \neq 0$.

Proof Applying 4.4.1, we have $H_{1}\left\{8 \iota_{6}, \nu_{6}, \eta_{9}\right\} \supseteq 64 \iota_{11} \circ \Delta^{\leftarrow}\left(\nu_{5} \circ \eta_{8}\right) \subseteq \pi_{11}\left(S^{11}\right)$. Since $11=$ $2 \times 5+1$ and 5 is odd the $E H \Delta$ sequence

$$
\ldots \rightarrow \pi_{10}\left(S^{5}\right) \stackrel{E}{\longrightarrow} \pi_{11}\left(S^{6}\right) \stackrel{H}{\longrightarrow} \pi_{11}\left(S^{11}\right) \stackrel{\Delta}{\longrightarrow} \pi_{9}\left(S^{5}\right) \rightarrow \ldots
$$

is exact and $\Delta^{\leftarrow}=\Delta^{-1}$. Moreover $\Delta \iota_{11}=\left[\iota_{5}, \iota_{5}\right]=\nu_{5} \circ \eta_{8}$ which is of order 2. It follows that $\Delta^{-1}\left(\nu_{5} \circ \eta_{8}\right)=\iota_{11}+2 \pi_{11}\left(S^{11}\right)$ and $H_{1}\left\{8 \iota_{6}, \nu_{6}, \eta_{9}\right\}=64 \iota+128 \pi_{11}\left(S^{11}\right)$. Since $H\left[\iota_{6}, \iota_{6}\right]=2 \iota_{11}$, we have $H 32\left[\iota_{6}, \iota_{6}\right]=64 \iota_{11}$. Since $\operatorname{kernel}(H)=E\left(\pi_{10}\left(S^{5}\right)\right)=0$, [T; Proposition 5.9], it follows that $32\left[\iota_{6}, \iota_{6}\right] \in{ }_{1}\left\{8 \iota_{6}, \nu_{6}, \eta_{9}\right\}$. Now the indeterminacy of the bracket is the subgroup

$$
E\left(\pi_{9}\left(S^{5}\right)\right) \circ \eta_{10}+\left(8 \iota_{6}\right) \circ \pi_{11}\left(S^{6}\right)=8 \iota_{6} \circ \pi_{11}\left(S^{6}\right) .
$$

But $H\left(8 \iota_{6} \circ \pi_{11}\left(S^{6}\right)\right)=64 H\left(\pi_{11}\left(S^{6}\right)\right)=128 \pi_{11}\left(S^{11}\right)$, [T; p.18]. Hence ${ }_{1}\left\{8 \iota_{6}, \nu_{6}, \eta_{9}\right\} \neq 0$, completing the proof.

\section{References}

[Bar] M.G. Barratt, Track groups I, Proc. London Math. Soc. (3) 5 (1955), 71-106.

[Bau] H.-J. Baues, Combinatorial Homotopy and 4-Dimensional Complexes, W. de Gruyter, Berlin, New York, 1981.

[BD] H.-J. Baues and W. Dreckmann, The cohomology of homotopy categories and the general linear group, K-theory 3 (1989), 307-338.

[Bé] J. Bénabou, Introduction to bicategories, Reports of the Midwest Category Seminar, Lecture Notes in Math. 47, Springer-Verlag, Berlin, 1967, pp. 1-77.

[Br1] R. Brown, Groupoids and van Kampen's theorem, Proc. London Math. Soc. (3) 17 (1967), 385-401.

[Br2] R. Brown, Elements of Modern Topology, McGraw-Hill, Maidenhead, 1968.

[BS] R. Brown and C.B. Spencer, Double groupoids and crossed modules, Cah. Top. Géom. Diff. 17 (1976), 343-362.

[E] C. Ehresmann, Catégories doubles et catégories structurées, C. R. A. S. Paris 256 (1963), 1198-1201.

[G] M. Grandis, Categorically algebraic foundations for homotopical algebra, Appl. Cat. Str. 5 (1997), 363-413.

[H] K.A. Hardie, Approximating the homotopy sequence of a pair of spaces, 
Tsukuba J. Math. 15 (1991), 85-98.

[HK] K.A. Hardie and K.H. Kamps, Secondary composition operations in homotopy pair theory, Tsukuba J. Math. 17 (1993), 201-220.

[HKK] K.A. Hardie, K.H. Kamps and R.W. Kieboom, A homotopy 2-groupoid of a Hausdorff space, App. Cat. Str. (to appear).

[J] I.M. James, Reduced product spaces, Ann. of Math. 82 (1955) , 170-197.

[KP] K.H. Kamps and T. Porter, Abstract Homotopy and Simple Homotopy Theory, World Scientific, Singapore, 1997.

[M] M. Mori, On higher Toda brackets, Bull. Coll. Sci. Univ. of the Ryukyus, No. 35 (1983), 1-4.

[R] J.W. Rutter, A homotopy classification of maps into an induced fibre space, Topology 6 (1967), 379-403.

[S] C.B. Spencer, An abstract setting for homotopy pushouts and pullbacks, Cah. Top. Géom. Diff. 18 (1977), 409-430.

[SW] C.B. Spencer and Y.L. Wong, Pullback and pushout squares in a special double category with connection, Cah. Top. Géom. Diff. 17 (1976), 343-362.

[T] H. Toda, Composition methods in homotopy groups of spheres, Ann. of Math. Studies 49, Princeton, 1962.

[W] G. Walker, Long Toda brackets, Proc. Adv. Study Inst. Alg. Top., August 10-23, 1970, Aarhus, Denmark.

The first author acknowledges support from the Foundation for Research Development and the University of Cape Town via the Topology and Category Theory Group.

This article may be accessed via WWW at http://www.rmi.acnet.ge/hha/ or by anonymous ftp at ftp://ftp.rmi.acnet.ge/pub/hha/volumes/1999/n4/n4.(dvi,ps,dvi.gz,ps.gz)

K.A. Hardie hardieka@iafrica.com

Department of Mathematics

University of Cape Town

7700 Rondebosch

South Africa

K.H. Kamps Heiner.Kamps@fernuni-hagen.de

Fachbereich Mathematik

Fernuniversität

Postfach 940

D-58084 Hagen

Germany

R.W. Kieboom rkieboom@vub.ac.be

Department of Mathematics

Vrije Universiteit Brussel

Pleinlaan 2, F 10

B-1050 Brussels

Belgium 